\title{
Purification of Murine Gammaherpesvirus 68 With Use of Differential Centrifugation
}

\author{
${ }^{1}$ Department of Microbiology and Virology, \\ Faculty of Natural Sciences, Comenius University in \\ Bratislava, Ilkovičova 6, Mlynská dolina, \\ 842 15, Bratislava, Slovak republic \\ 2Slovak Academy of Sciences, Biomedical Research \\ Center, Institute of Virology, Dúbravská cesta 9, \\ 84505 Bratislava, Slovak republic \\ ${ }^{3}$ Department of Galenic Pharmacy, Faculty of Pharmacy, \\ Comenius University in Bratislava, Odbojar \\ 1083232 Bratislava 3, Slovak Republic
}

Abstract The method for separation of viral particles in a concentrated form from the environment is called virus purification. Viruses are required to be purified for a range of studies in which it is necessary to distinguish the properties or structure of a virus from the host cells or culture media, including analysis of viral polypeptide structures and membrane glycoprotein function. Our objective was to purify murine gammaherpesvirus 68 (MHV-68, MuHV-4) using the centrifuge, equipment and other materials available in our laboratory. After infection of baby hamster kidney 21 (BHK-21) cells with MHV-68 with the multiplicity of infection (MI) of 0.01 and following virus multiplication, we repeatedly froze and thawed the cell culture to disrupt the cells and release the virus particles into the culture medium. We used low-speed centrifugation ( $3000 \mathrm{rpm}$ at $4^{\circ} \mathrm{C}$ ) to separate the viral particles from cell debris. Subsequently, we transferred the supernatant containing virus particles to a fresh centrifuge tube and centrifuged at a speed of $8000 \mathrm{rpm}(8801 \mathrm{~g})$ and $11,000 \mathrm{rpm}(=16,639 \mathrm{~g})$ and at $4^{\circ} \mathrm{C}$. We tested different centrifugation durations of $2,4,6$ and 8 hours. To evaluate the quality of the obtained purified MHV-68 virus by this method and compare it to purified MHV-68 sample acquired by conventional ultracentrifugation on sucrose cushion (30\%, w/v), we used the SDS-PAGE separation method using a $4 \%-20 \%(\mathrm{w} / \mathrm{v})$ and 6\%-14\% (w/v) gradient gel. We obtained the best results with 6-hour-long centrifugation at 11,000 rpm. In conclusion, we managed to optimise virus purification method using the equipment available in our laboratory and prepared purified MHV-68 virus in sufficient concentration for determination of MHV-68 virus proteins.

Keywords MHV-68-purification-virus proteins-SDS-PAGE

\section{INTRODUCTION}

The aim of the virus purification method is to separate it from all particles of cellular origin and culture media (Mistríková et al., 2016). Viruses need to be purified for many studies in which it is necessary to distinguish the properties or structure of a virus from the properties or structure of host cells (or culture media), such as analysis of the structure of viral polypeptides or the function of membrane glycoproteins (Killington et al., 1996). Differential (ultra)centrifugation (Fig. 1) is a common technique used to purify particles of defined size (i.e., virions). It is based on differences in the sedimentation rate of particles in suspension, which depends not only on their size, density and morphology, but also on the properties of the medium in which the virions are located and the force applied during centrifugation (Killington et al., 1996; Taulbe and Furst, 2005).
By using a density gradient centrifugation technique (particle separation is achieved by density gradient sedimentation), it is possible to achieve a more accurate separation of biological particles of similar dimensions but different densities. A large number of gradient materials are available, such as various dextrans and sucrose, or commercially available, for example, Percoll, Ficoll and others (Taulbe and Furst, 2005). The aim of our experimental work in the presented paper was to prepare purified murine gammaherpesvirus 68 (MHV-68, MuHV-4) propagated on the stabilised baby hamster kidney 21 (BHK21) cell line and use it for further research. Using laboratory instrumentation, ultracentrifuge and other necessary materials available in our laboratory, we performed and optimised the MHV-68 purification method. 
a)

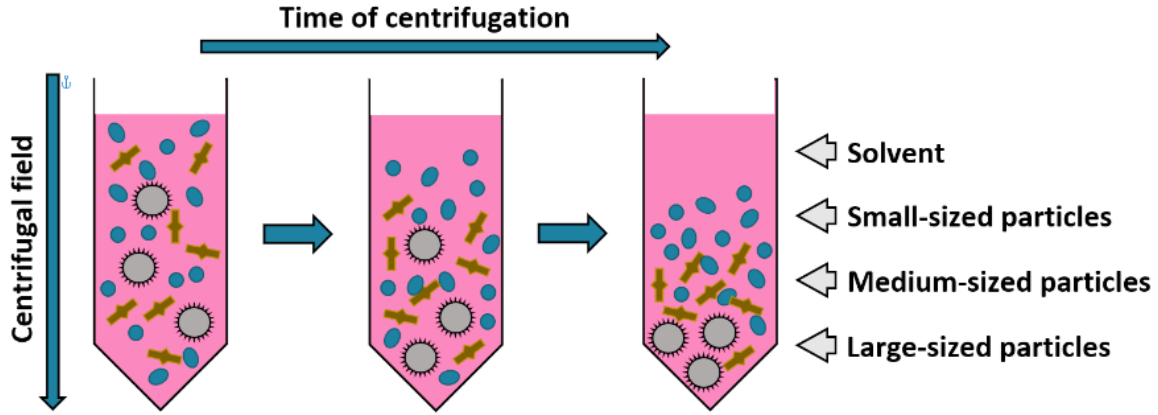

b)

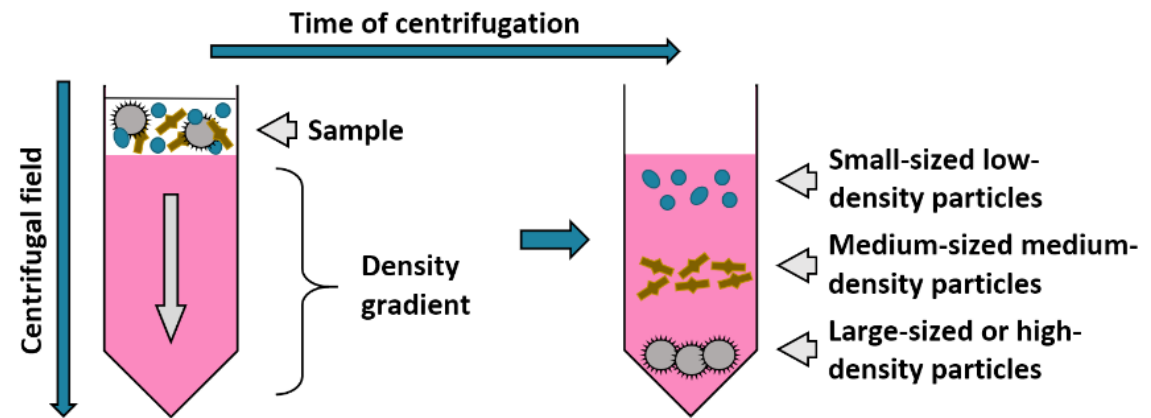

Figure 1. Diagram of particle behaviour during differential centrifugation and density gradient centrifugation. a) During the differential centrifugation of a suspension of particles in a centrifugal field, the movement of the particles depends on their density, shape and size. b) To separate the biological particles by means of a density gradient, the samples are layered on the prepared gradient before centrifugation. To achieve separation, the sample is centrifuged, until the isopycnic position for the desired particles in the gradient is achieved.

\section{MATERIALS AND METHODS}

We used baby hamster kidney 21 (BHK-21) cells to propagate MHV-68 virus. Cells were cultured in Dulbecco's Modified Eagle Medium (DMEM) medium (Lonza, Belgium) supplemented with $7 \%$ fetal bovine serum (FBS; Sigma Aldrich, USA), 1\% PSA (antibiotics - $100 \mathrm{U} / \mathrm{ml}$ penicillin-streptomycin and $100 \mu \mathrm{g} / \mathrm{ml}$ amphotericin) and $1 \%$ glutamine (Sigma Aldrich, USA) at $37^{\circ} \mathrm{C}$ in the presence of $5 \% \mathrm{CO}^{2}$. After virus adsorption, we replaced DMEM with $7 \%$ FBS with DMEM with $2 \%$ FBS. Cells were infected with MHV-68 virus at a multiplicity of infection of 0.1 and cultured until a cytopathic effect was developed. The virus/cell suspension was frozen and thawed 3 times $\left(-80^{\circ} \mathrm{C}\right)$. We transferred the virus-medium solution from the culture flasks to $50-\mathrm{ml}$ centrifuge tubes and centrifuged for $30 \mathrm{~min}$ at $2000 \mathrm{rpm}$ at $4^{\circ} \mathrm{C}$ (low-speed centrifugation to remove cell debris). After centrifugation, we transferred the supernatant to a new centrifuge tube and centrifuged. We tested four different durations of 2, 4, 6 and 8 hours and two different centrifugation speeds of $8000 \mathrm{rpm}(8801 \mathrm{~g})$ and 11,000 rpm $(16,639 \mathrm{~g})$ at $4^{\circ} \mathrm{C}$ in an Eppendorf $5804 \mathrm{R}$ centrifuge with an FA-45-6-30 rotor - primary high-speed centrifugation. After this centrifugation, we removed the supernatant, resuspended the pellet in $25 \mathrm{ml}$ phosphate-buffered saline (PBS) and centrifuged for 2 hours at 11,000 rpm $(16,639 \mathrm{~g})$ at $4^{\circ} \mathrm{C}$ (secondary high-speed centrifugation) in an Eppendorf $5804 \mathrm{R}$ centrifuge with an FA-45-6-30 rotor. After removing the supernatant, we resuspended the virus pellet in $1 \mathrm{ml}$ PBS, filled into microtubes in volume of $100 \mu \mathrm{l}$ and froze at $-80^{\circ} \mathrm{C}$. The purification process is schematically shown in Fig. 2.

To evaluate the quality of the obtained samples, we used the sodium dodecyl sulphate-polyacrylamide gel electrophoresis (SDS-PAGE) separation method using a gradient gel. To create a gradient separating gel, we prepared two bis-acrylamide solutions with different densities: one with the lowest gradient density ( $4 \% \mathrm{w} / \mathrm{v}$ and $6 \% \mathrm{w} / \mathrm{v})$ and the second one with the highest gradient density $(20 \% \mathrm{w} / \mathrm{v}$ and $16 \% \mathrm{w} / \mathrm{v})$ Following this, we drew up the lower density solution $(4 \%$ $\mathrm{w} / \mathrm{v}$ and $6 \% \mathrm{w} / \mathrm{v}$ ) into a serological pipette and then a higher density solution ( $20 \% \mathrm{w} / \mathrm{v}$ and $16 \% \mathrm{w} / \mathrm{v})$ in the ratio of $1: 1$. The total volume must correspond to the volume of the gel cast (BioRad mini-PROTEAN gel cast has a volume of $5 \mathrm{ml}$ ). Next, we drew up approximately $1 \mathrm{ml}$ of air into the serological pipette containing gel solutions and allowed the air bubble to ascend to the surface. The air bubble travel mixes the solution and forms a density gradient within pipette. Lastly, we applied the solution to the cast evenly and overlaid it with $99.6 \% \mathrm{w} / \mathrm{v}$ isopropanol and let it polymerise.

\section{RESULTS AND DISCUSSION}

For viral protein separation, we used gradient SDS-PAGE in the density range of $4 \%-20 \%(\mathrm{w} / \mathrm{v})$ and $6 \%-16 \%(\mathrm{w} / \mathrm{v})$, 


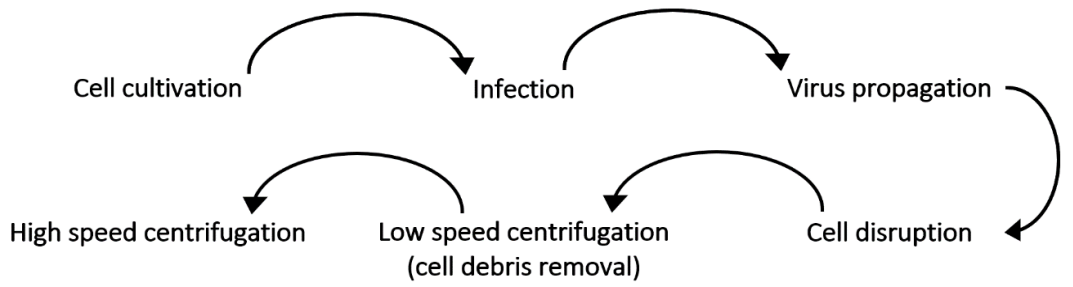

Figure 2. Schematic diagram of virus purification steps.

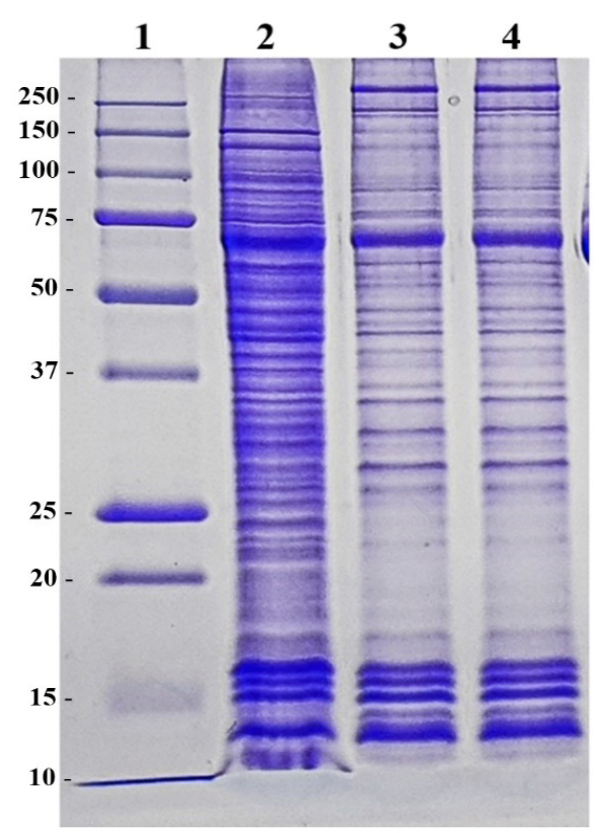

Figure 3. Polypeptide profile of purified MHV-68 in gradient SDSPAGE (6\%-14\%, w/v). Lane 1: ladder (Precision Plus Protein ${ }^{\mathrm{mm}}$ Dual Xtra); lane 2: purified MHV-68 (centrifuged 6 hours) - $10 \mu \mathrm{l}$ lane; lane 3: purified MHV-68 (centrifuged 6 hours) - $5 \mu$ l/lane; lane 4: purified MHV-68 (centrifuged 6 hours) $-3 \mu$ I/lane.

considering the large range of molecular weights of the individual MHV-68 proteins (from 15 to $240 \mathrm{kDa}$ ). Samples obtained by centrifugation at $8000 \mathrm{rpm}$ were unsuitable for further analysis due to insufficient concentration of MHV-68 proteins (data not shown). We obtained improved results by increasing the centrifugation speed to 11,000 rpm. Centrifugation of 2 and 4 hours was not sufficient to concentrate the virus in the pellet (data not shown). By extending the centrifugation interval to 6 hours, we obtained MHV-68 purification at a sufficient concentration, resulting in a polypeptide profile of viral proteins of specific size. We applied purified MHV-68 after 6-hour-long centrifugation at $11,000 \mathrm{rpm}$ in various volumes on gradient SDS-PAGE with a density range of $6 \%-14 \%(w / v)$ (Fig. 3). Protein profile of purified murine gammaherpesvirus obtained by ultracentrifugation on sucrose cushion (Reichel et al., 1991) was comparable to MHV-68 purified by our method (after 6-hour-long centrifugation at 11,000 rpm).

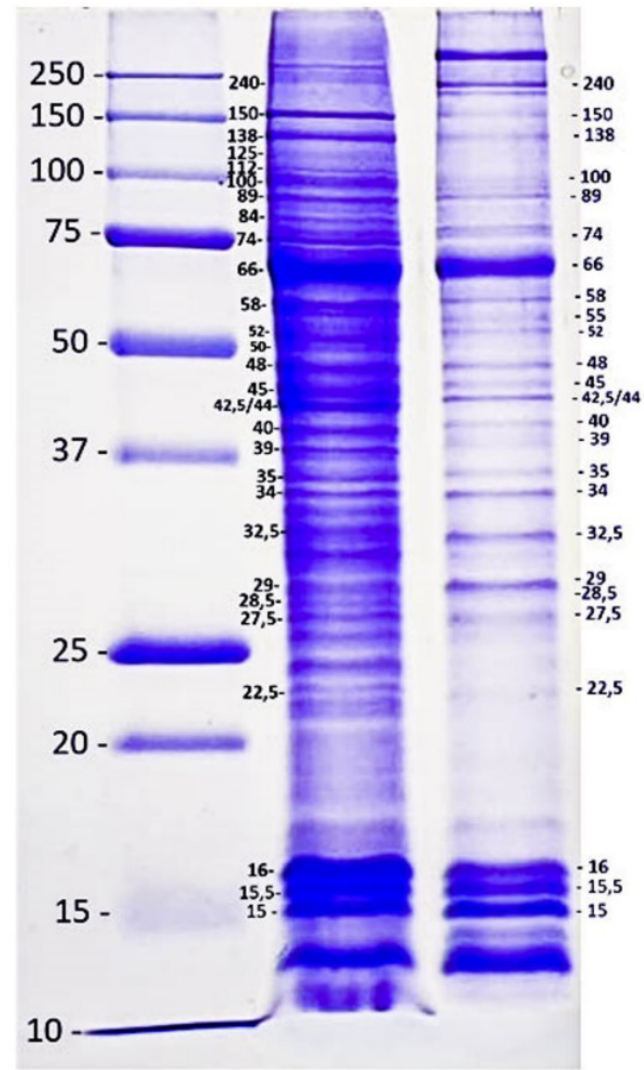

Figure 4. Molecular weights (in $\mathrm{kDa}$ ) of viral proteins of purified MHV-68, gradient SDS-PAGE (6\%-14\% w/v). Lane 1: ladder (Precision Plus Protein ${ }^{m}$ Dual Xtra); lane 2: purified MHV-68 (centrifuged 6 hours) - $12 \mu \mathrm{l} /$ lane; lane 3: purified MHV-68 (centrifuged 6 hours) $-6 \mu$ l/lane.

The next step was to determine the approximate molecular weight (in $\mathrm{kDa}$ ) of the viral proteins of the obtained purified MHV-68. Fig. 4 shows in more detail the protein profile of the obtained purified MHV-68. We determined the molecular weight of individual proteins according to Reichel et al. (1991). In conclusion, we were able to prepare purified MHV-68 virus and determine the approximate molecular weight of viral proteins using available instruments and methods in our laboratory. We obtained the best results with primary centrifugation duration of 6 hours at a centrifugation speed of $11,000 \mathrm{rpm}(16,639 \mathrm{~g})$ at $4^{\circ} \mathrm{C}$ (in our case, 6 hours of primary and 2 hours of secondary high-speed centrifugation was sufficient to purify MHV-68 virus). 


\section{Purification of Murine Gammaherpesvirus 68 With Use of Differential Centrifugation}

\section{ACKNOWLEDGMENT}

This research was carried out with the financial support from VEGA $1 / 0061 / 18$ and UK/240/2021.

\section{References}

[1] Mistríková M., Stančeková M., Kostrábová A. (2016) Základné laboratórne techniky používané vo virológii. 3. prepracované vydanie, Bratislava, Univerzita Komenského, strany 39-41, ISBN 9788022342087

[2] Killington R.A., Stokes A., Hierholzer J.C. (1996) 4-Virus purification. In: Mahy B.W.J. (ed.), Kangro H.O. (ed.), Virology Methods Manual, Academic Press, strany 71-89, ISBN 9780124653306

[3] Taulbee D.N., Furst A. (2005) - Centrifugation | Preparative. In: Worsfold P. (ed.), Townshend A. (ed.), Poole C (ed.), Encyclopedia of Analytical Science (Second Edition), Elsevier, strany 469-481, ISBN 9780123693976

[4] Reichel M, Matis J, Lesso J. Stancekova, M. (1991). Polypeptides synthesized in rabbit cells infected with murine herpesvirus (MHV): a comparison of proteins specified by various MHV strains. Acta virologiea 35, 268275 